\title{
Modified Approach for Optimization of Real Life Transportation Problem in Neutrosophic Environment
}

\author{
Akanksha Singh, ${ }^{1}$ Amit Kumar, ${ }^{1}$ and S. S. Appadoo ${ }^{2}$ \\ ${ }^{1}$ School of Mathematics, Thapar University, Patiala, Punjab, India \\ ${ }^{2}$ Department of Supply and Chain Management, University of Manitoba, Winnipeg, MB, Canada \\ Correspondence should be addressed to S. S. Appadoo; appadoo@cc.umanitoba.ca
}

Received 11 April 2017; Revised 13 July 2017; Accepted 16 July 2017; Published 22 August 2017

Academic Editor: Erik Cuevas

Copyright (c) 2017 Akanksha Singh et al. This is an open access article distributed under the Creative Commons Attribution License, which permits unrestricted use, distribution, and reproduction in any medium, provided the original work is properly cited.

\begin{abstract}
To the best of our knowledge, there is only one approach for solving neutrosophic cost minimization transportation problems. Since neutrosophic transportation problems are a new area of research, other researchers may be attracted to extend this approach for solving other types of neutrosophic transportation problems like neutrosophic solid transportation problems, neutrosophic time minimization transportation problems, neutrosophic transshipment problems, and so on. However, after a deep study of the existing approach, it is noticed that a mathematical incorrect assumption has been used in these existing approaches; therefore there is a need to modify these existing approaches. Keeping the same in mind, in this paper, the existing approach is modified. Furthermore, the exact results of some existing transportation problems are obtained by the modified approach.
\end{abstract}

\section{Introduction}

In daily life problems several times there is a need to transport the product from various sources to different destinations. To find a way to transport the product in such a manner so that the total transportation cost is minimum is called the optimal way and the problem is called cost minimization transportation problems [1]. Different methods have been proposed in the literature to find the optimal way of such cost minimization transportation problems in which cost for transporting unit quantity of the product, availability of the product at the sources, and demand of the product at the destinations are represented as real numbers.

However, to assume these parameters as real numbers is not always valid according to real life situations; for example, the transportation cost depends upon the circumstances like price of petrol/diesel, weather, travel time, traffic jam, and so on. Similarly, availability of crops varies according to the monsoon, fertilizers, chemicals, and so on; demands of the various clothes depend on the season, fashion trends, discount offers, and so on. Furthermore, the opinions of the experts about these parameters indicate that they cannot always be represented as real numbers; for example, generally experts provide their opinion about these parameters in terms of linguistic variables like high, very high, low, very low, and so on.

Being one of the widely adopted ways in the literature, to deal with such situations is to represent these parameters as fuzzy numbers [2] and its extensions [3]. Thamaraiselvi and Santhi [4] pointed out that neutrosophic set [5], one of the extensions of fuzzy set, is used in different research areas. However, till now no one has used the neutrosophic set in transportation problems, while several researchers have used fuzzy numbers for representing various parameters of transportation problems [6-14]. Therefore, Thamaraiselvi and Santhi [4] proposed the approaches for solving neutrosophic transportation problem of Type I (transportation problem in which cost for transporting unit quantity of the product is represented as trapezoidal neutrosophic number, whereas availability and demands are represented as real numbers) and neutrosophic transportation problem of Type II (transportation problem in which cost for transporting unit quantity of the product, availability of a product, and demand of the product are represented as trapezoidal neutrosophic numbers). 
Since neutrosophic transportation problems are new area of research, others may be attracted to extend these approaches for solving other types of neutrosophic transportation problems like neutrosophic solid transportation problems, neutrosophic time minimization transportation problems, neutrosophic transshipment problems, and so on. However, after a deep study of these existing approaches, it is noticed that a mathematical incorrect assumption has been used in these existing approaches; therefore there is a need to modify these existing approaches. Keeping the same in mind, in this paper, these existing approaches are modified. Furthermore, the exact results of some existing transportation problems are obtained by the modified approaches.

\section{A Brief Review of Thamaraiselvi and Santhi Approaches}

To point out the mathematical incorrect assumptions in the approaches, proposed by Thamaraiselvi and Santhi [4], there is a need to describe these approaches. Therefore, in this section, a brief review of the approaches, proposed by the Thamaraiselvi and Santhi [4], for solving both types of neutrosophic transportation problems is discussed in a brief manner.

2.1. Thamaraiselvi and Santhi Approach for Solving Neutrosophic Transportation Problem of Type I. Using the approach, proposed by Thamaraiselvi and Santhi [4], the optimal solution of a neutrosophic transportation problem of Type I can be obtained as follows.

Step 1. Formulate the neutrosophic transportation problem as a neutrosophic linear programming problem $\left(P_{1}\right)$.

$$
\begin{array}{ll}
\text { Minimize } & \left(\sum_{i=0}^{m} \sum_{j=0}^{n} \widetilde{c}_{i j}^{N} x_{i j}\right) \\
\text { Subject to } & \sum_{j=0}^{n} x_{i j}=a_{i}, \quad i=1,2, \ldots, m, \\
& \sum_{i=0}^{n} x_{i j}=b_{j}, \quad j=1,2, \ldots, n, \\
& x_{i j} \geq 0, \quad \forall i, j,
\end{array}
$$

where

$x_{i j}$ is the number of units of the product transported from $i$ th source to $j$ th destination,

$\tilde{c}_{i j}^{N}$ is the neutrosophic cost of one unit quantity transported from $i$ th source to $j$ th destination,

$a_{i}$ is the total availability of the product at the source $i$,

$b_{j}$ is the total demand of the product at the destination j.
Step 2. Transform the neutrosophic linear programming problem $\left(P_{1}\right)$ into its equivalent crisp linear programming problem $\left(P_{2}\right)$.

$$
\text { Minimize } S\left(\sum_{i=0}^{m} \sum_{j=0}^{n} \widetilde{c}_{i j}^{N} x_{i j}\right)
$$

Subject to Constraints of problem $\left(P_{1}\right)$,

where

$$
\begin{aligned}
& S\left(\widetilde{a}^{N}\right)=S\left(\left\langle\left(a_{1}, a_{2}, a_{3}, a_{4}\right), \mu_{\tilde{a}}, \nu_{\widetilde{a}}, \lambda_{\tilde{a}}\right\rangle\right) \\
& \quad=\frac{1}{16}\left[a_{1}+a_{2}+a_{3}+a_{4}\right]\left[\mu_{\tilde{a}}+\left(1-v_{\tilde{a}}\right)+\left(1-\lambda_{\tilde{a}}\right)\right] .
\end{aligned}
$$

Step 3. Transform the crisp linear programming problem $\left(P_{2}\right)$ into its equivalent crisp linear programming problem $\left(P_{3}\right)$.

$$
\operatorname{Minimize}\left(\sum_{i=1}^{m} \sum_{j=1}^{n} S\left(\widetilde{c}_{i j}^{N}\right) x_{i j}\right)
$$

Subject to Constraints of problem $\left(P_{1}\right)$.

Step 4. Represent the crisp linear programming problem $\left(P_{3}\right)$ into tabular form shown in Table 1.

Step 5. Find the crisp optimal solutions $\left\{x_{i j}\right\}$ of crisp transportation problem presented in Table 1.

Step 6. Find the total minimum neutrosophic transportation cost by putting the optimal solution $\left\{x_{i j}\right\}$, obtained from Step 5 , in $\sum_{i=1}^{m} \sum_{j=1}^{n} \widetilde{c}_{i j}^{N} x_{i j}$.

2.2. Thamaraiselvi and Santhi Approach for Solving Neutrosophic Transportation Problem of Type II. Using the approach, proposed by Thamaraiselvi and Santhi [4], the optimal solution of a neutrosophic transportation problem of Type II can be obtained as follows.

Step 1. Formulate the neutrosophic transportation problem as a neutrosophic linear programming problem $\left(P_{4}\right)$.

$$
\begin{array}{ll}
\text { Minimize } & \left(\sum_{i=0}^{m} \sum_{j=0}^{n} \widetilde{c}_{i j}^{N} \widetilde{x}_{i j}^{N}\right) \\
\text { Subject to } & \sum_{j=0}^{n} \widetilde{x}_{i j}^{N}=\widetilde{a}_{i}^{N}, \quad i=1,2, \ldots, m, \\
& \sum_{i=0}^{m} \widetilde{x}_{i j}^{N}=\widetilde{b}_{j}^{N}, \quad j=1,2, \ldots, n, \\
& \widetilde{x}_{i j}^{N} \geq 0, \quad \forall i, j .
\end{array}
$$


TABLE 1: Tabular representation of transformed crisp transportation problem.

\begin{tabular}{lcccccc}
\hline Sources & \multicolumn{5}{c}{ Destinations } & Supply \\
\hline$S_{1}$ & $D_{1}$ & $D_{2}$ & $D_{3}$ & $\ldots$ & $D_{n}$ & $a_{1}$ \\
$S_{2}$ & $S\left(\widetilde{c}_{11}^{N}\right)$ & $S\left(\widetilde{c}_{12}^{N}\right)$ & $S\left(\widetilde{c}_{13}^{N}\right)$ & $\ldots$ & $S\left(\widetilde{c}_{1 n}^{N}\right)$ & $a_{2}$ \\
$S_{3}$ & $S\left(\widetilde{c}_{21}^{N}\right)$ & $S\left(\widetilde{c}_{22}^{N}\right)$ & $S\left(\widetilde{c}_{23}^{N}\right)$ & $\ldots$ & $\left.a_{3}\right)$ & $\vdots$ \\
$\vdots$ & $S\left(\widetilde{c}_{31}^{N}\right)$ & $S\left(\widetilde{c}_{32}^{N}\right)$ & $S\left(\widetilde{c}_{33}^{N}\right)$ & $\ldots$ & $\vdots$ & $\left.a_{m}\right)$ \\
$S_{m}$ & $\vdots$ & $\vdots$ & $\vdots$ & $\vdots$ & $S\left(\widetilde{c}_{m n}^{N}\right)$ & $b_{n}$ \\
\hline Demand & $S\left(\widetilde{c}_{m 1}^{N}\right)$ & $S\left(\widetilde{c}_{m 2}^{N}\right)$ & $S\left(\widetilde{c}_{m 3}^{N}\right)$ & $\ldots$ & & \\
\hline
\end{tabular}

TABLE 2: Tabular representation of neutrosophic transportation problem of Type II.

\begin{tabular}{|c|c|c|c|c|c|c|}
\hline \multirow{2}{*}{ Sources } & \multicolumn{5}{|c|}{ Destinations } & \multirow{2}{*}{ Supply } \\
\hline & $D_{1}$ & $D_{2}$ & $D_{3}$ & $\ldots$ & $D_{n}$ & \\
\hline$S_{1}$ & $S\left(\widetilde{c}_{11}^{N}\right)$ & $S\left(\widetilde{c}_{12}^{N}\right)$ & $S\left(\widetilde{c}_{13}^{N}\right)$ & $\ldots$ & $S\left(\widetilde{c}_{1 n}^{N}\right)$ & $\tilde{a}_{1}^{N}$ \\
\hline$S_{2}$ & $S\left(\widetilde{c}_{21}^{N}\right)$ & $S\left(\widetilde{c}_{22}^{N}\right)$ & $S\left(\widetilde{c}_{23}^{N}\right)$ & $\ldots$ & $S\left(\widetilde{c}_{2 n}^{N}\right)$ & $\tilde{a}_{2}^{N}$ \\
\hline$S_{3}$ & $S\left(\widetilde{c}_{31}^{N}\right)$ & $S\left(\widetilde{c}_{32}^{N}\right)$ & $S\left(\widetilde{c}_{33}^{N}\right)$ & $\ldots$ & $S\left(\widetilde{c}_{3 n}^{N}\right)$ & $\tilde{a}_{3}^{N}$ \\
\hline$\vdots$ & $\vdots$ & 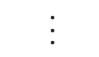 & $\vdots$ & $\vdots$ & $\vdots$ & $\vdots$ \\
\hline$S_{m}$ & $S\left(\widetilde{c}_{m 1}^{N}\right)$ & $S\left(\widetilde{c}_{m 2}^{N}\right)$ & $S\left(\widetilde{c}_{m 3}^{N}\right)$ & $\ldots$ & $S\left(\widetilde{c}_{m n}^{N}\right)$ & $\tilde{a}_{m}^{N}$ \\
\hline Demand & $\tilde{b}_{1}^{N}$ & $\widetilde{b}_{2}^{N}$ & $\widetilde{b}_{3}^{N}$ & $\ldots$ & $\widetilde{b}_{n}^{N}$ & \\
\hline
\end{tabular}

Step 2. Transform the neutrosophic linear programming problem $\left(P_{4}\right)$ into its equivalent neutrosophic linear programming problem $\left(P_{5}\right)$.

$$
\text { Minimize } S\left(\sum_{i=1}^{m} \sum_{j=1}^{n} \widetilde{c}_{i j}^{N} \widetilde{x}_{i j}^{N}\right)
$$

Subject to Constraints of problem $\left(P_{4}\right)$.

Step 3. Transform the neutrosophic linear programming problem $\left(P_{5}\right)$ into its equivalent neutrosophic linear programming problem $\left(P_{6}\right)$.

$$
\text { Minimize } \sum_{i=1}^{m} \sum_{j=1}^{n} S\left(\widetilde{c}_{i j}^{N}\right) \widetilde{x}_{i j}^{N}
$$

Subject to Constraints of problem $\left(P_{4}\right)$.

Step 4. Represent the neutrosophic linear programming problem $\left(P_{6}\right)$ into tabular form shown in Table 2.

Step 5. Find the neutrosophic optimal solution $\left\{\tilde{x}_{i j}^{N}\right\}$ of transportation problem presented in Table 2 .

Step 6. Find the total minimum neutrosophic transportation cost by putting the optimal solution $\left\{\tilde{x}_{i j}^{N}\right\}$, obtained in Step 3, in $\sum_{i=1}^{m} \sum_{j=1}^{n} \widetilde{c}_{i j}^{N} \tilde{x}_{i j}^{N}$.

\section{Mathematical Incorrect Assumption Considered in Thamaraiselvi and Santhi Approaches}

In this section, the mathematical incorrect assumption, considered in Thamaraiselvi and Santhi approaches [4], is pointed out.

It is obvious from Steps 2 and 3 of the first approach, presented in Section 2.1, that Thamaraiselvi and Santhi [4] have assumed that the neutrosophic linear programming problem $\left(P_{2}\right)$ can be transformed into neutrosophic linear programming problem $\left(P_{3}\right)$. Similarly, it is obvious from Steps 2 and 3 of the second approach, presented in Section 2.2, that Thamaraiselvi and Santhi [4] have assumed that the neutrosophic linear programming problem $\left(P_{5}\right)$ can be transformed into neutrosophic linear programming problem $\left(P_{6}\right)$.

It is pertinent to mention that, to transform the problem $\left(P_{2}\right)$ into $\left(P_{3}\right)$ as well as $\left(P_{5}\right)$ into $\left(P_{6}\right)$, Thamaraiselvi and Santhi [4] have considered that $\left(\sum_{i=0}^{m} \sum_{j=0}^{n} \widetilde{c}_{i j}^{N} x_{i j}\right)=\sum_{i=1}^{m} \sum_{j=1}^{n} S\left(\widetilde{c}_{i j}^{N}\right) x_{i j}$; that is, Thamaraiselvi and Santhi [4] have considered the assumption that if $\widetilde{a}^{N}=\left\langle\left(a_{1}, a_{2}, a_{3}, a_{4}\right), \mu_{\tilde{a}}, \nu_{\widetilde{a}}, \lambda_{\widetilde{a}}\right\rangle$ and $\widetilde{b}^{N}=\left\langle\left(b_{1}, b_{2}\right.\right.$, $\left.\left.b_{3}, b_{4}\right), \mu_{\tilde{a}}, \nu_{\widetilde{a}}, \lambda_{\widetilde{a}}\right\rangle$ are two trapezoidal neutrosophic numbers, then

$$
S\left(\widetilde{a}^{N}+\widetilde{b}^{N}\right)=S\left(\widetilde{a}^{N}\right)+S\left(\widetilde{b}^{N}\right) .
$$

However, the following example clearly indicates that

$$
S\left(\widetilde{a}^{N}+\widetilde{b}^{N}\right) \neq S\left(\widetilde{a}^{N}\right)+S\left(\widetilde{b}^{N}\right) .
$$


Let $\widetilde{a}^{N}=\langle(2,4,6,8) ; 0.1,0.2,0.3\rangle$ and $\tilde{b}^{N}=\langle(3,6,9$, $12) ; 0.4,0.5,0.6\rangle$ be two trapezoidal neutrosophic numbers; then according to existing result [4, Def. 10, pp. 4]

$$
\begin{aligned}
\widetilde{a}^{N}+\widetilde{b}^{N}= & \langle(2,4,6,8) ; 0.1,0.2,0.3\rangle \\
& +\langle(3,6,9,12) ; 0.4,0.5,0.6\rangle \\
= & \langle(5,10,15,20) ; 0.1,0.5,0.6\rangle .
\end{aligned}
$$

Furthermore, using the existing result [4, Def. 11, pp. 4]

$$
\begin{aligned}
S\left(\widetilde{a}^{N}+\widetilde{b}^{N}\right) & =\frac{1}{16}(5+10+15+20)(0.1+0.5+0.4) \\
& =3.125
\end{aligned}
$$

while

$$
\begin{aligned}
S\left(\tilde{a}^{N}\right)+S\left(\widetilde{b}^{N}\right) \\
=\left\{\frac{1}{16}(2+4+6+8)(0.1+0.8+0.7)\right\} \\
+\quad\left\{\frac{1}{16}(3+6+9+12)(0.4+0.5+0.4)\right\} \\
=2+2.4375=4.4375 .
\end{aligned}
$$

It is obvious that

$$
S\left(\widetilde{a}^{N}+\tilde{b}^{N}\right) \neq S\left(\widetilde{a}^{N}\right)+S\left(\widetilde{b}^{N}\right) .
$$

Hence, the approaches for solving neutrosophic transportation problem, proposed by Thamaraiselvi and Santhi [4], are not valid.

\section{An important Result}

It is obvious from Section 3 that, to modify the existing approaches [4], there is a need to find the exact relation between $S\left(\sum_{i=1}^{n} a_{i}\right)$ and $\sum_{i=1}^{n} S\left(a_{i}\right)$. Therefore, in this section, the exact relation between $S\left(\sum_{i=1}^{n} a_{i}\right)$ and $\sum_{i=1}^{n} S\left(a_{i}\right)$ is obtained.

Let $\tilde{a}^{N}=\left\langle\left(a_{1}, a_{2}, a_{3}, a_{4}\right), \mu_{\tilde{a}}, \nu_{\tilde{a}}, \lambda_{\tilde{a}}\right\rangle$ and $\tilde{b}^{N}=\left\langle\left(b_{1}\right.\right.$, $\left.\left.b_{2}, b_{3}, b_{4}\right), \mu_{\tilde{a}}, \nu_{\tilde{a}}, \lambda_{\tilde{a}}\right\rangle$ be two trapezoidal neutrosophic numbers; then using the existing arithmetic operations [4, Def. 10, pp. 4] $\sum_{i=1}^{n} \tilde{a}^{N}=\left\langle\left(\sum_{i=1}^{n} a_{i 1}, \sum_{i=1}^{n} a_{i 2}, \sum_{i=1}^{n} a_{i 3}\right.\right.$, $\left.\left.\sum_{i=1}^{n} a_{i 4}\right) ; \min _{1 \leq i \leq n}\left(\mu_{a_{i}}\right), \max _{1 \leq i \leq n}\left(\nu_{a_{i}}\right), \max _{1 \leq i \leq n}\left(\lambda_{a_{i}}\right)\right\rangle$, and using the existing results $[4$, Def. 12, pp. 4$]$,

$$
\begin{aligned}
& S\left(\sum_{i=1}^{n} \widetilde{a}^{N}\right)=S\left\langle\left(\sum_{i=1}^{n} a_{i 1}, \sum_{i=1}^{n} a_{i 2}, \sum_{i=1}^{n} a_{i 3}, \sum_{i=1}^{n} a_{i 4}\right) ;\right. \\
& \left.\min _{1 \leq i \leq n}\left(\mu_{a_{i}}\right), \max _{1 \leq i \leq n}\left(v_{a_{i}}\right), \max _{1 \leq i \leq n}\left(\lambda_{a_{i}}\right)\right\rangle=\frac{1}{16}\left(\sum_{i=1}^{n} a_{i 1}\right. \\
& \left.+\sum_{i=1}^{n} a_{i 2}+\sum_{i=1}^{n} a_{i 3}+\sum_{i=1}^{n} a_{i 4}\right)\left[\min _{1 \leq i \leq n}\left(\mu_{a_{i}}\right)\right. \\
& \left.+\left(1-\max _{1 \leq i \leq n}\left(v_{a_{i}}\right)+\right)\left(1-\max _{1 \leq i \leq n}\left(\lambda_{a_{i}}\right)\right)\right] .
\end{aligned}
$$

Furthermore, using the existing results [4, Def. 12, pp. 4],

$$
\begin{aligned}
S\left(a_{i}\right)= & \frac{1}{16}\left(a_{i 1}+a_{i 2}+a_{i 3}+a_{i 4}\right) \\
& \cdot\left[\mu_{a_{i}}+\left(1-v_{a_{i}}\right)+\left(1-\lambda_{a_{i}}\right)\right] .
\end{aligned}
$$

From (8) and (9),

$$
\begin{aligned}
& S\left(\sum_{i=1}^{n} \widetilde{a}^{N}\right) \\
& =\left(\min _{1 \leq i \leq n}\left(\mu_{a_{i}}\right)+\max _{1 \leq i \leq n}\left(1-v_{a_{i}}\right)+\max _{1 \leq i \leq n}\left(1-\lambda_{a_{i}}\right)\right) \\
& \quad \cdot \sum_{i=1}^{n}\left(\frac{S\left(\tilde{a}_{i}^{N}\right)}{\mu_{a_{i}}+\left(1-v_{a_{i}}\right)+\left(1-\lambda_{a_{i}}\right)}\right) .
\end{aligned}
$$

\section{Modified Approach for Solving Neutrosophic Transportation Problems}

In this section, to resolve the flaws of the existing approaches, pointed out in Section 3, the existing approaches [4] are modified.

5.1. Modified Approaches for Solving Neutrosophic Transportation Problem of Type I. In this section, the existing approach is modified to solve neutrosophic transportation problem of Type I.

The steps of the modified approach are as follows.

Step 1. Use Steps 1 and 2 of the existing approach [4] to obtain the problem $\left(P_{2}\right)$.

Step 2. Using the relation, obtained in Section 4, transform the problem $\left(P_{2}\right)$ into its equivalent neutrosophic linear programming problem $\left(P_{7}\right)$.

$$
\operatorname{Minimize}\left(\sum_{i=1}^{m} \sum_{j=1}^{n} M\left(\widetilde{c}_{i j}^{N}\right) x_{i j}\right)
$$

Subject to Constraints of problem $\left(P_{1}\right)$,

where

$$
\begin{aligned}
M & \left(\widetilde{c}_{i j}^{N}\right) \\
= & \left(\min _{1 \leq i \leq n}\left(\mu_{\widetilde{c}_{i j}^{N}}\right)+\max _{1 \leq i \leq n}\left(1-\nu_{\widetilde{c}_{i j}^{N}}\right)+\max _{1 \leq i \leq n}\left(1-\lambda_{\widetilde{c}_{i j}^{N}}\right)\right) \\
& \cdot \sum_{i=1}^{m} \sum_{j=1}^{n}\left(\frac{S\left(\widetilde{c}_{i j}^{N} x_{i j}\right)}{\mu_{\widetilde{c}_{i j}^{N}}+\left(1-\nu_{\widetilde{i}_{i j}^{N}}\right)+\left(1-\lambda_{\widetilde{c}_{i j}^{N}}\right)}\right) .
\end{aligned}
$$

Step 3. Represent the neutrosophic linear programming problem $\left(P_{7}\right)$ into tabular form as shown in Table 3.

Step 4. Find the crisp optimal solutions $\left\{x_{i j}\right\}$ of crisp transportation problem presented in Table 3. 
TABLE 3: Tabular representation of transformed crisp transportation problem.

\begin{tabular}{|c|c|c|c|c|c|c|}
\hline \multirow{2}{*}{ Sources } & \multicolumn{5}{|c|}{ Destinations } & \multirow{2}{*}{ Supply } \\
\hline & $D_{1}$ & $D_{2}$ & $D_{3}$ & $\ldots$ & $D_{n}$ & \\
\hline$S_{1}$ & $M\left(\widetilde{c}_{11}^{N}\right)$ & $M\left(\widetilde{c}_{12}^{N}\right)$ & $M\left(\widetilde{c}_{13}^{N}\right)$ & $\ldots$ & $M\left(\widetilde{c}_{1 n}^{N}\right)$ & $a_{1}$ \\
\hline$S_{2}$ & $M\left(\left(_{21}^{N}\right)\right.$ & $M\left(\left(_{22}^{N}\right)\right.$ & $M\left(\widetilde{c}_{23}^{N}\right)$ & $\ldots$ & $M\left(\widetilde{c}_{2 n}^{N}\right)$ & $a_{2}$ \\
\hline$S_{3}$ & $M\left(\left(^{N}\right)_{31}^{N}\right)$ & $M\left(\tilde{c}_{32}^{N}\right)$ & $M\left(\widetilde{c}_{33}^{N}\right)$ & $\ldots$ & $M\left(\widetilde{c}_{3 n}^{N}\right)$ & $a_{3}$ \\
\hline$\vdots$ & $\vdots$ & $\vdots$ & $\vdots$ & $\vdots$ & $\vdots$ & $\vdots$ \\
\hline$S_{m}$ & $M\left(\widetilde{c}_{m 1}^{N}\right)$ & $M\left(\widetilde{c}_{m 2}^{N}\right)$ & $M\left(\widetilde{c}_{m 3}^{N}\right)$ & $\ldots$ & $M\left(\widetilde{c}_{m n}^{N}\right)$ & $a_{m}$ \\
\hline Demand & $b_{1}$ & $b_{2}$ & $b_{3}$ & $\ldots$ & $b_{n}$ & \\
\hline
\end{tabular}

Step 5. Find the total minimum neutrosophic transportation cost by putting the optimal solution $\left\{x_{i j}\right\}$, obtained from Step 4 , in $\sum_{i=1}^{m} \sum_{j=1}^{n} \widetilde{c}_{i j}^{N} x_{i j}$.

5.2. Modified Approach for Solving Neutrosophic Transportation Problem of Type II. In this section, the existing approach is modified to solve neutrosophic transportation problem of Type II.

The steps of the modified approach are as follows.

Step 1. Use Steps 1 and 2 of the existing approach to obtain problem $\left(P_{5}\right)$.

Step 2. Using the relation, obtained in Section 4, transform the problem $\left(P_{5}\right)$ into problem $\left(P_{8}\right)$

$$
\text { Minimize } S\left(\sum_{i=1}^{m} \sum_{j=1}^{n} M\left(\widetilde{c}_{i j}^{N}\right) \widetilde{x}_{i j}^{N}\right)
$$

Subject to Constraints of problem $\left(P_{4}\right)$.

Step 3. Represent the neutrosophic linear programming problem $\left(P_{8}\right)$ into tabular form as shown in Table 4.

Step 4. Find the neutrosophic optimal solution $\left\{\tilde{x}_{i j}^{N}\right\}$ of neutrosophic transportation problem presented in Table 4.

Step 5. Find the total minimum neutrosophic transportation cost by putting the optimal solution $\left\{\tilde{x}_{i j}^{N}\right\}$, obtained in Step 4, in $\sum_{i=1}^{m} \sum_{j=1}^{n} \widetilde{c}_{i j}^{N} \tilde{x}_{i j}^{N}$.

\section{Exact Solution of Numerical Problems}

Thamaraiselvi and Santhi [4] solved a neutrosophic transportation problem of Type I and Type II to illustrate their proposed approaches. However as discussed in Section 3 that Thamaraiselvi and Santhi [4] have used some mathematical incorrect assumptions in their proposed approaches, the optimal solution of these problems, obtained by Thamaraiselvi and Santhi [4], is not exact. In this section, the exact solution of these problems is obtained by modified approaches.

6.1. Exact Solution of Neutrosophic Transportation Problem of Type I. Thamaraiselvi and Santhi [4] solved the neutrosophic transportation problem of Type I, presented in Table 5, by their proposed approach.
In this section the exact solution of this problem is obtained by the modified approach. Using the modified approach the exact solution of neutrosophic transportation problem of Type I, presented in Table 5, can be obtained as follows.

Step 1. Using Steps 1 to 3 of the modified approach the neutrosophic transportation problem, presented in Table 5, can be transformed into crisp transportation problem (presented in Table 6).

Step 2. When solving the crisp transportation problem (Table 6), the obtained optimal solution is

$$
\begin{aligned}
& x_{12}=23, \\
& x_{14}=3, \\
& x_{21}=17, \\
& x_{24}=7, \\
& x_{33}=28, \\
& x_{34}=2 .
\end{aligned}
$$

Step 3. Using the optimal solution, the minimum total neutrosophic cost is

$$
\begin{aligned}
& \operatorname{Minimize}\left(\widetilde{Z}^{N}=\sum_{i=0}^{3} \sum_{j=0}^{4} x_{i j} \widetilde{c}_{i j}^{N}\right) \\
&= 23\langle(5,8,10,14) ; 0.3,0.8,0.7\rangle \\
&+3\langle(14,17,21,28) ; 0.3,0.8,0.7\rangle \\
&+17\langle(0,1,3,6) ; 0.3,0.8,0.7\rangle \\
&+7\langle(9,11,14,16) ; 0.3,0.8,0.7\rangle \\
&+28\langle(5,7,8,10) ; 0.3,0.8,0.7\rangle \\
&+2\langle(5,9,14,19) ; 0.3,0.8,0.7\rangle \\
&=\langle(370,543,694,938) ; 0.3,0.8,0.7\rangle .
\end{aligned}
$$

6.2. Exact Solution of Neutrosophic Transportation Problem of Type II. Thamaraiselvi and Santhi [4] solved the neutrosophic transportation problem of Type II, presented in Table 7, by their proposed approach. 
TABLE 4: Tabular representation of neutrosophic transportation problem of Type II.

\begin{tabular}{|c|c|c|c|c|c|c|}
\hline \multirow{2}{*}{ Sources } & \multicolumn{5}{|c|}{ Destinations } & \multirow{2}{*}{ Supply } \\
\hline & $D_{1}$ & $D_{2}$ & $D_{3}$ & $\ldots$ & $D_{n}$ & \\
\hline$S_{1}$ & $M\left(\widetilde{c}_{11}^{N}\right)$ & $M\left(\widetilde{c}_{12}^{N}\right)$ & $M\left(\widetilde{c}_{13}^{N}\right)$ & $\ldots$ & $M\left(\widetilde{c}_{1 n}^{N}\right)$ & $\tilde{a}_{1}^{N}$ \\
\hline$S_{2}$ & $M\left(\widetilde{c}_{21}^{N}\right)$ & $M\left(\widetilde{c}_{22}^{N}\right)$ & $M\left(\widetilde{c}_{23}^{N}\right)$ & $\ldots$ & $M\left(\widetilde{c}_{2 n}^{N}\right)$ & $\widetilde{a}_{2}^{N}$ \\
\hline$S_{3}$ & $M\left(\widetilde{c}_{31}^{N}\right)$ & $M\left(\widetilde{c}_{32}^{N}\right)$ & $M\left(\widetilde{c}_{33}^{N}\right)$ & $\ldots$ & $M\left(\widetilde{c}_{3 n}^{N}\right)$ & $\tilde{a}_{3}^{N}$ \\
\hline$\vdots$ & $\vdots$ & $\vdots$ & $\vdots$ & $\vdots$ & $\vdots$ & $\vdots$ \\
\hline$S_{m}$ & $M\left(\widetilde{c}_{m 1}^{N}\right)$ & $M\left(\widetilde{c}_{m 2}^{N}\right)$ & $M\left(\widetilde{c}_{m 3}^{N}\right)$ & $\ldots$ & $M\left(\widetilde{c}_{m n}^{N}\right)$ & $\tilde{a}_{m}^{N}$ \\
\hline Demand & $\widetilde{b}_{1}^{N}$ & $\widetilde{b}_{2}^{N}$ & $\tilde{b}_{3}^{N}$ & $\ldots$ & $\tilde{b}_{n}^{N}$ & \\
\hline
\end{tabular}

TABLE 5: Input data for neutrosophic transportation problem.

\begin{tabular}{lccccc}
\hline & $D 1$ & $D 2$ & $D 3$ & $D$ & Supply \\
\hline O1 & $\langle(3,5,6,8) ; 0.6,0.5,0.4\rangle$ & $\langle(5,8,10,14) ; 0.3,0.6,0.6\rangle$ & $\langle(12,15,19,22) ; 0.6,0.4,0.5\rangle$ & $\langle(14,17,21,28 ; 0.8,0.2,0.6)\rangle$ & 26 \\
O2 & $\langle(0,1,3,6) ; 0.7,0.5,0.3\rangle$ & $\langle(5,7,9,11) ; 0.9,0.7,0.5\rangle$ & $\langle(15,17,19,22) ; 0.4,0.8,0.4\rangle$ & $\langle(9,11,14,16) ; 0.5,0.4,0.7\rangle$ & 24 \\
O3 & $\langle(4,8,11,15) ; 0.6,0.3,0.2\rangle$ & $\langle(1,3,4,6) ; 0.6,0.3,0.5\rangle$ & $\langle(5,7,8,10) ; 0.5,0.4,0.7\rangle$ & $\langle(5,9,14,19) ; 0.3,0.7,0.6\rangle$ & 30 \\
\hline Demand & 17 & 23 & 28 & 12 & \\
\hline
\end{tabular}

TABLE 6: Crisp transportation problem.

\begin{tabular}{lccccc}
\hline & $D 1$ & $D 2$ & $D 3$ & $D 4$ & Supply \\
\hline O1 & 3 & 4 & 8 & 9 & 26 \\
O2 & 1 & 4 & 8 & 6 & 24 \\
O3 & 4 & 2 & 3 & 5 & 30 \\
\hline Demand & 17 & 23 & 28 & 12 & \\
\hline
\end{tabular}

In this section, the exact solution of this problem is obtained by the modified approach. Using the modified approach the exact solution of neutrosophic transportation problem of Type II, presented in Table 7, can be obtained as follows.

Step 1. Using Steps 1 to 3 of the modified approach the neutrosophic transportation problem presented in Table 7 can be transformed into neutrosophic transportation problem (presented in Table 8).

Step 2. When solving the neutrosophic transportation problem with crisp cost (Table 8), the obtained optimal solution is

$$
\begin{aligned}
& \tilde{x}_{11}^{N}=\langle(-6,2,7,11) ; 0.7,0.3,0.4\rangle, \\
& \tilde{x}_{12}^{N}=\langle(17,21,24,28) ; 0.8,0.2,0.4\rangle, \\
& \tilde{x}_{21}^{N}=\langle(2,9,16,27) ; 0.5,0.5,0.6\rangle, \\
& \widetilde{x}_{24}^{N}=\langle(-10,6,18,29) ; 0.5,0.5,0.6\rangle, \\
& \tilde{x}_{33}^{N}=\langle(24,29,32,35) ; 0.9,0.5,0.3\rangle, \\
& \widetilde{x}_{34}^{N}=\langle(-14,-4,3,13) ; 0.8,0.5,0.4\rangle .
\end{aligned}
$$

Step 3. Using the optimal solution the minimum total neutrosophic cost is

$$
\begin{aligned}
\operatorname{Minimize} & \left(\widetilde{Z}^{N}=\sum_{i=0}^{3} \sum_{j=0}^{4} \widetilde{x}_{i j}^{N} \widetilde{c}_{i j}^{N}\right) \\
= & \langle(-6,2,7,11) ; 0.7,0.3,0.4\rangle \\
& \times\langle(3,5,6,8) ; 0.6,0.5,0.4\rangle \\
& +\langle(17,21,24,28) ; 0.8,0.2,0.4\rangle \\
& \times\langle(5,8,10,14) ; 0.3,0.6,0.6\rangle \\
& +\langle(2,9,16,27) ; 0.5,0.5,0.6\rangle \\
& \times\langle(0,1,3,6) ; 0.7,0.5,0.3\rangle \\
& +\langle(-10,6,18,29) ; 0.5,0.5,0.6\rangle \\
& \times\langle(9,11,14,16) ; 0.5,0.4,0.7\rangle \\
& +\langle(24,29,32,35) ; 0.9,0.5,0.3\rangle \\
& \times\langle(5,7,8,10) ; 0.5,0.4,0.7\rangle \\
& +\langle(-14,-4,3,13) ; 0.8,0.5,0.4\rangle \\
& \times\langle(5,9,14,19) ; 0.3,0.7,0.6\rangle \\
= & \langle(27,420,880,1703) ; 0.3,0.7,0.7\rangle .
\end{aligned}
$$




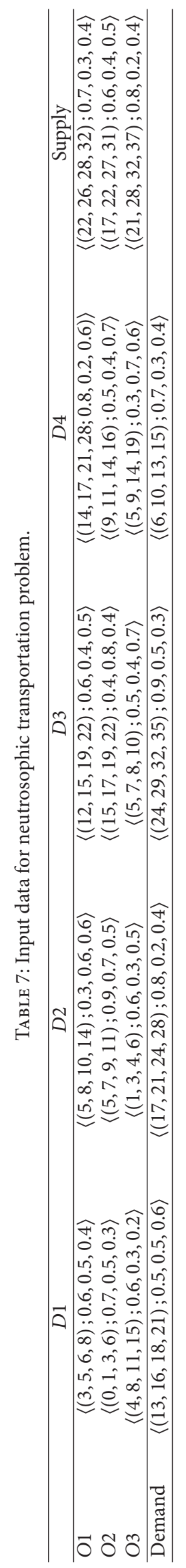




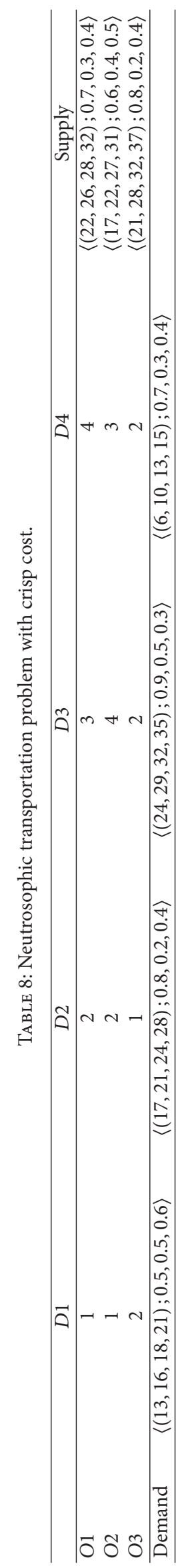


TABLE 9: Existing and modified score values.

Minimum score value of neutrosophic transportation cost

Existing approaches [4]

Modified approaches

First problem

140.118

127.25

Second problem

182.981

170.437

\section{Comparative Study}

The score value of minimized neutrosophic transportation cost, obtained by existing approaches [4] and modified approaches, is shown in Table 9.

It is obvious from the results, shown in Table 9, that the score value of minimum neutrosophic transportation cost, obtained by modified approach, is less than the score value of the minimum value of neutrosophic transportation cost obtained by the existing approach [4]. This clearly indicates that the optimal solution obtained by the modified approach is better than the neutrosophic transportation cost by existing approach [4].

\section{Conclusion}

It is pointed out that it is not genuine to use the existing approaches [4] as in these approaches mathematical incorrect assumption has been used. Therefore, the existing approaches [4] are modified. Furthermore, the exact solution of existing neutrosophic transportation problem is obtained by the modified approach.

\section{Conflicts of Interest}

The authors declare that there are no conflicts of interest regarding the publication of this paper.

\section{References}

[1] F. L. Hitchcock, "The distribution of a product from several sources to numerous localities," Journal of Mathematics and Physics, vol. 20, pp. 224-230, 1941.

[2] L. A. Zadeh, "Fuzzy sets," Information and Control, vol. 8, no. 3, pp. 338-353, 1965.

[3] H. Bustince, E. Barrenechea, M. Pagola et al., "A historical account of types of fuzzy sets and their relationships," IEEE Transactions on Fuzzy Systems, vol. 24, no. 1, pp. 179-194, 2016.

[4] A. Thamaraiselvi and R. Santhi, "A new approach for optimization of real life transportation problem in neutrosophic environment," Mathematical Problems in Engineering, vol. 2016, Article ID 5950747, 9 pages, 2016.

[5] F. Smarandache, "Neutrosophic set, a generalization of the intuitionistic fuzzy set," International Journal of Pure and Applied Mathematics, vol. 24, no. 3, pp. 287-297, 2005.

[6] M. ÓhÉigeartaigh, "A fuzzy transportation algorithm," Fuzzy Sets and Systems, vol. 8, no. 3, pp. 235-243, 1982.

[7] S. Chanas and D. Kuchta, "A concept of the optimal solution of the transportation problem with fuzzy cost coefficients," Fuzzy Sets and Systems. An International Journal in Information Science and Engineering, vol. 82, no. 3, pp. 299-305, 1996.
[8] F. Jiménez and J. L. Verdegay, "Solving fuzzy solid transportation problems by an evolutionary algorithm based parametric approach," European Journal of Operational Research, vol. 117, no. 3, pp. 485-510, 1999.

[9] A. Kaur and A. Kumar, "A new method for solving fuzzy transportation problems using ranking function," Applied Mathematical Modelling. Simulation and Computation for Engineering and Environmental Systems, vol. 35, no. 12, pp. 5652-5661, 2011.

[10] A. Kaur and A. Kumar, "A new approach for solving fuzzy transportation problems using generalized trapezoidal fuzzy numbers," Applied Soft Computing Journal, vol. 12, no. 3, pp. 1201-1213, 2012.

[11] A. Nagoor Gani and S. Abbas, "Solving intuitionistic fuzzy transportation problem using zero suffix algorithm," International Journal of Mathematical Sciences and Engineering Applications. IJMSEA, vol. 6, no. 3, pp. 73-82, 2012.

[12] G. Gupta, J. Kaur, and A. Kumar, "A note on 'fully fuzzy fixed charge multi-item solid transportation problem,' Applied Soft Computing Journal, vol. 41, pp. 418-419, 2016.

[13] S. K. Sidhu, A. Kumar, and A. Kaur, "A note on 'A fuzzy approach to transport optimization problem,' Optimization and Engineering, vol. 17, no. 4, pp. 987-992, 2016.

[14] D. Rani, T. R. Gulati, and A. Kumar, "A method for unbalanced transportation problems in fuzzy environment," Sadhana Academy Proceedings in Engineering Sciences, vol. 39, no. 3, pp. 573-581, 2014. 


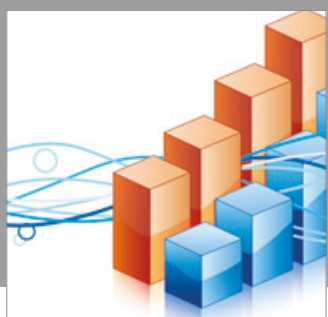

Advances in

Operations Research

vatersals

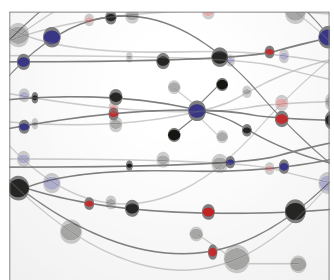

\section{The Scientific} World Journal
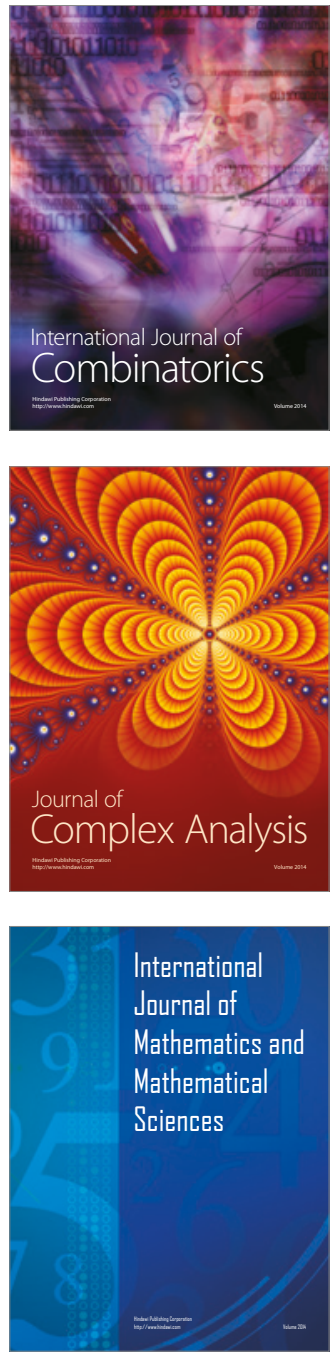
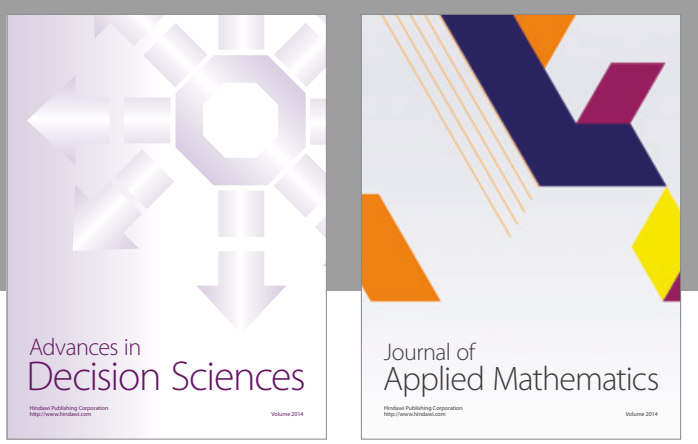

Algebra

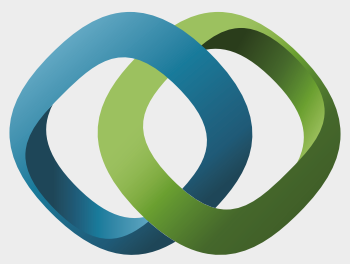

\section{Hindawi}

Submit your manuscripts at

https://www.hindawi.com
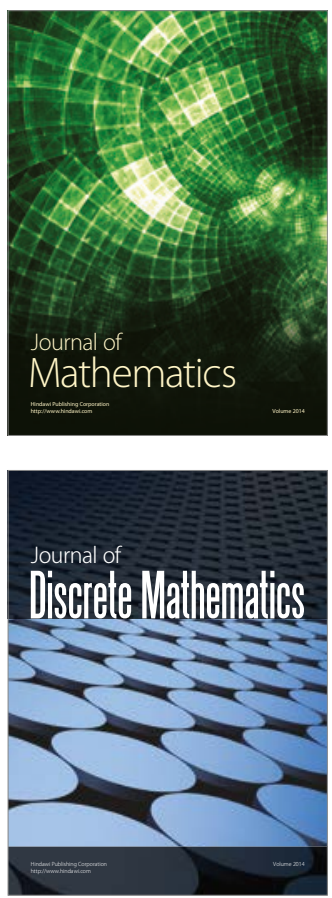

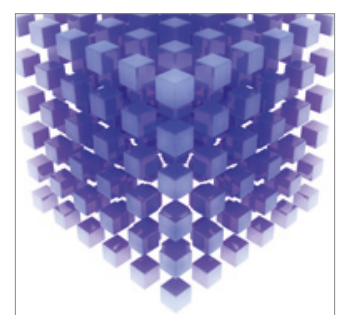

Mathematical Problems in Engineering
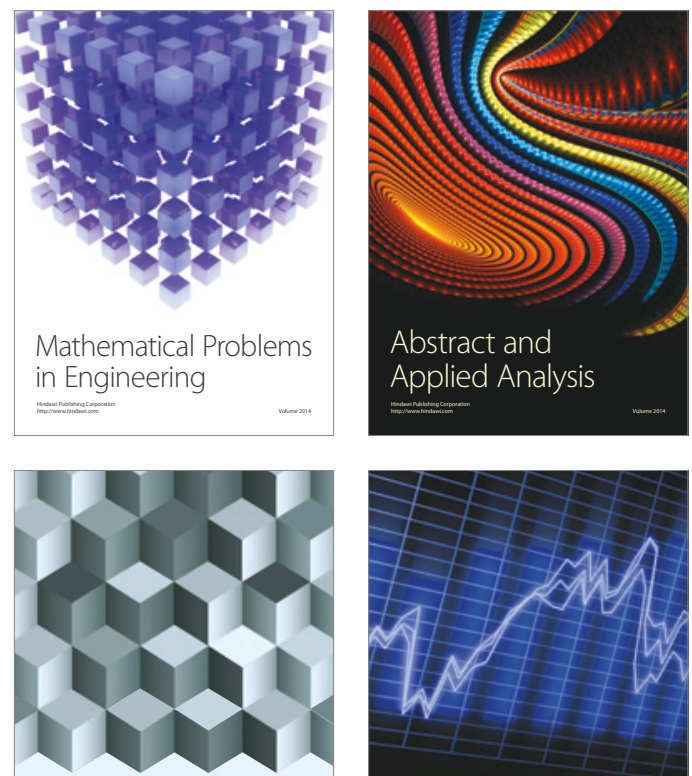

Journal of

Function Spaces

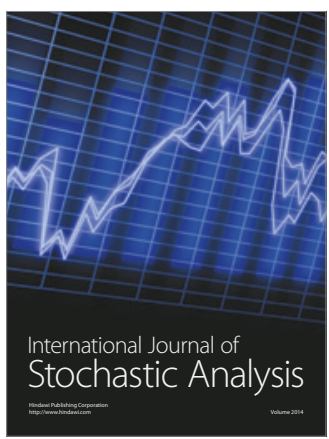

Probability and Statistics
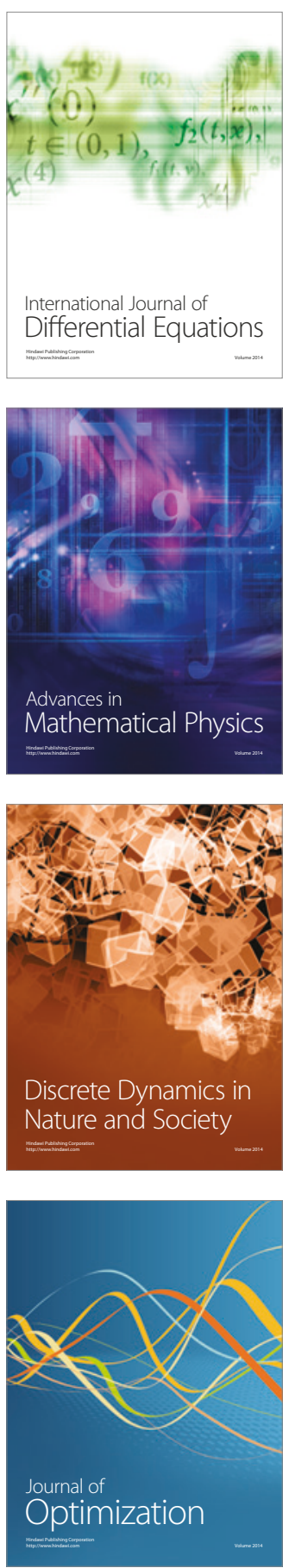